\title{
Focus Issue: CNMAC 2014
}

\author{
Igor L. Freire ${ }^{1}$ • Paulo F. A. Mancera ${ }^{2}$
}

Received: 9 February 2016 / Accepted: 23 February 2016 / Published online: 4 March 2016

(C) SBMAC - Sociedade Brasileira de Matemática Aplicada e Computacional 2016

The Brazilian Society of Computational and Applied Mathematics (SBMAC) was founded on November 1, 1978. Since then it has been organizing its most important and biggest scientific meeting: the National Congress of Computational and Applied Mathematics (CNMAC). This annual congress has around 800 participants, including under-graduate and graduate students, researchers, lecturers and professionals from public services and industries. SBMAC prizes and honors are also awarded during CNMAC. In 2014, CNMAC took place in Natal, Rio Grande do Norte, and there the SBMAC Boarding decided to organize a special number of the journal Computational and Applied Mathematics by inviting some authors to submit manuscripts connected to the contributed papers presented in the congress.

The present issue of Computational and Applied Mathematics contains a selection of refereed papers covering recent developments presented during the event. They are concerned with:

- fractional calculus, where the authors discuss modeling using Caputo fractional derivative and analyse an unexpected behavior of this derivative;

- numerical studies of incompressible fluid using regularization of Navier-Stokes equations;

- numerical simulation of two-dimensional incompressible turbulent flows;

- stable maps between closed orientable surfaces;

- inverse methodology for estimating chlorophyll- $a$ concentration in waters.

Communicated by Eduardo Souza de Cursi.

Igor L. Freire

igor.freire@ufabc.edu.br; igor.leite.freire@gmail.com

Paulo F. A. Mancera

pmancera@ibb.unesp.br

1 Centro de Matemática, Computação e Cognição, Universidade Federal do ABC, Avenida dos Estados, 5001, Santo André, SP 09210-280, Brazil

2 IBB, Departamento de Bioestatística, Universidade Estadual Paulista "Júlio de Mesquita Filho", Distrito de Rubião Junior, Botucatu, SP 18618-689, Brazil 
We would like to thank all authors who submitted their papers for this issue. We are also indebted with the anonymous referees for their hard and precious work. Special thank is due to José Eduardo Souza de Cursi, Editor-in-Chief of Computational and Applied Mathematics, for his prompt help and support. We are also pleased in thanking SBMAC Boarding for trusting us this Focus Issue. 